\title{
Characterization of novel polymorphic genomic microsatellite markers of Boehmeria tricuspis (Hance) Makino
}

\author{
Q. Tang, J.H. Chen, G.G. Zang and M.B. Luan \\ Key Laboratory of Stem-fiber Biomass and Engineering Microbiology, \\ Institute of Bast Fiber Crops, Chinese Academy of Agricultural Sciences, \\ Ministry of Agriculture, Changsha, China \\ Corresponding author: M.B. Luan \\ E-mail: luanmingbao2002@126.com
}

Genet. Mol. Res. 15 (2): gmr. 15027882

Received October 22, 2015

Accepted January 18, 2016

Published April 26, 2016

DOI http://dx.doi.org/10.4238/gmr.15027882

\begin{abstract}
In the present study, 59 polymorphic microsatellite loci of Boehmeria tricuspis (Hance) Makino were developed from the specific length amplified fragment sequencing data library of genome. The number of alleles per locus ranged from two to five, and the observed and expected heterozygosities ranged from 0.0000 to 1.0000 and from 0.0769 to 0.6751 , respectively. Among the 59 loci, 25 displayed significant deviations from Hardy-Weinberg expectations $(\mathrm{P}<0.05)$. The developed simple sequence repeat markers should be useful for studying population genetics in B. tricuspis (Hance) Makino, for providing further knowledge on its population differentiation, breeding system, and dispersal ability, as well as quantitative trait locus mapping. These markers could also be valuable genetic resources for closely related species.
\end{abstract}

Key words: Boehmeria tricuspis (Hance) Makino; SSR; SLAF 


\section{INTRODUCTION}

Boehmeria tricuspis (Hance) Makino, which is naturally distributed in China, Japan, and Korea, has traditionally been used for fabric production. Presently, it is also used as a material for apomictic research in the field of basic science (Chen et al., 2011b). However, in the past decade, natural populations of B. tricuspis (Hance) Makino have decreased, with some populations having disappeared, due to human activities as well as climatic and environmental changes. Therefore, it is essential to protect natural resources of B. tricuspis (Hance) Makino. For its long-term sustenance, it is necessary to establish adequate management plans for the conservation of this species with a good understanding of its genetic diversity, population structure, and genetic differentiation. Simple sequence repeats (SSRs) are significantly effective in species conservation and management because of their high polymorphism, abundance, codominance, and small length (Chen et al., 2011a). To date, no SSR markers have been reported in B. tricuspis (Hance) Makino. Therefore, development of SSR markers is imperative. In the present paper, we report 59 novel genomic SSR loci, developed through the specific length amplified fragment sequencing (SLAF) data of the genome.

\section{MATERIAL AND METHODS}

From the SLAF data library of B. tricuspis (Hance) Makino genome, 80 sequences, containing mono-, di-, tri-, tetra-, penta-, or hexanucleotide units repeated at least 15, 8, 5, 4, 3, or 3 times, respectively, were selected to evaluate the polymorphism. The SSR markers were designed with primer 5 software (Lalitha, 2000).

The major parameters used for primer design were according to Chen et al. (2011a). The primers were synthesized by BioAsia Biotech (Shanghai, China). Young leaves of 30 individuals were used for DNA extraction following the protocol of Tiangen DNA extraction kit (Beijing, China). Polymerase chain reactions were carried out in 10- $\mu \mathrm{L}$ reaction volumes with $1 \mathrm{X}$ PCR buffer, $0.2 \mathrm{mM}$ dNTP, $1 \mathrm{U}$ Taq DNA polymerase (Tiangen), $0.5 \mu \mathrm{M}$ each primer, and $0.5 \mu \mathrm{L}$ DNA under the following PCR conditions: $5 \mathrm{~min}$ at $94^{\circ} \mathrm{C}$, followed by 30 cycles of $30 \mathrm{~s}$ at $95^{\circ} \mathrm{C}, 30 \mathrm{~s}$ at the primer-specific annealing temperature, $30 \mathrm{~s}$ at $72^{\circ} \mathrm{C}$, and a final extension of $10 \mathrm{~min}$ at $72^{\circ} \mathrm{C}$. The PCR products were separated on $8 \%$ polyacrylamide gels by electrophoresis, and stained using a silver dye according to Zhang et al. (2000). Observed heterozygosity $\left(H_{\mathrm{O}}\right)$, expected heterozygosity $\left(H_{\mathrm{E}}\right)$, and significant deviations from Hardy-Weinberg equilibrium (HWE) were estimated using Popgen1.32.

\section{RESULTS AND DISCUSSION}

Details of the developed microsatellite loci and their variability characteristics across 30 individuals of a natural population are summarized in Table 1. In total, 59 of 156 loci were successfully amplified and demonstrated to be polymorphic. The number of alleles per locus ranged from two to five and the observed and expected heterozygosities from 0.0000 to 1.0000 and from 0.0769 to 0.6751 , respectively. Of the 59 loci, 25 displayed significant deviations from Hardy-Weinberg expectations $(\mathrm{P}<0.05)$. 
Table 1. Characterization of 59 microsatellite loci of Boehmeria tricuspis (Hance) Makino, described by locus name, repeat motif, forward $(\mathrm{F})$ and reverse $(\mathrm{R})$ primer sequences, size of allele (Size), optimal annealing temperatures $(\mathrm{Ta})$, number of alleles $\left(N_{\mathrm{A}}\right)$, observed heterozygosity $\left(H_{\mathrm{O}}\right)$, expected heterozygosity $\left(H_{\mathrm{E}}\right)$, and significance of departure from Hardy-Weinberg equilibrium (HWE).

\begin{tabular}{|c|c|c|c|c|c|c|c|c|}
\hline Locus & Repeat & Primer sequences $\left(5^{\prime}-3^{\prime}\right)$ & Size (bp) & $\mathrm{Ta}\left({ }^{\circ} \mathrm{C}\right)$ & $N_{\mathrm{A}}$ & $H_{\mathrm{O}}$ & $H_{\mathrm{E}}$ & HWE P value \\
\hline N1 & $(\mathrm{T})_{19}$ & $\begin{array}{l}\text { F: TGATTCCGTGTTGTGTTGTTC } \\
\text { R: CCCGAAATTATGCGAACTGT }\end{array}$ & 130 & 59 & 2 & 0.1875 & 0.1754 & 0.74 \\
\hline N2 & $(\mathrm{T})_{18}$ & $\begin{array}{l}\text { F: TGCATGAGCTCTGATCTTCCT } \\
\text { R: TTTGGTCTGTTAAGACTCAGAACTGT }\end{array}$ & 117 & 60 & 2 & 0.3215 & 0.3992 & 0.30 \\
\hline N3 & $(\mathrm{A})_{15}$ & $\begin{array}{l}\text { F: TGAGCAAGGTTTTTGACGTTC } \\
\text { R: TGCAACTCCGAGATGAAAAA }\end{array}$ & 139 & 59 & 2 & 0.0000 & 0.5116 & 0.00 \\
\hline N4 & $(\mathrm{AG})_{10}$ & $\begin{array}{l}\text { F: AGACCCTCCGTTTCAGAGGT } \\
\text { R: CACCGCAGCAAAACTCTACA }\end{array}$ & 106 & 60 & 3 & 0.1796 & 0.2282 & 0.43 \\
\hline N5 & $(\mathrm{AG}) 9$ & $\begin{array}{l}\text { F: TCTCACCAGCCAAACAAAGA } \\
\text { R: GATTCCTCTGCTCGCTCATC }\end{array}$ & 148 & 59 & 2 & 0.0000 & 0.4249 & 0.00 \\
\hline N6 & $(\mathrm{TC})_{10}$ & $\begin{array}{l}\text { F: TTCTCACCGTTAATCTCGGC } \\
\text { R: AATCTCTGGACTAATATTCTGTGATTC }\end{array}$ & 113 & 57 & 3 & 0.3333 & 0.4723 & 0.01 \\
\hline N7 & $(\mathrm{GA})_{8}$ & $\begin{array}{l}\text { F: TCACACAGTAAGACAAAGAGAAAGG } \\
\text { R: CGTAGCCAAAATGTCCTTCAA }\end{array}$ & 137 & 59 & 2 & 0.0000 & 0.5085 & 0.00 \\
\hline N8 & $(\mathrm{AG}) 9$ & $\begin{array}{l}\text { F: ATGGCTTTGATGGGATTGAA } \\
\text { R: CTGTCTCTCACACGCACACC }\end{array}$ & 113 & 60 & 2 & 0.0769 & 0.0769 & 1.00 \\
\hline N9 & $(\mathrm{TAA})_{5}$ & $\begin{array}{l}\text { F: TGGGAAAATGAATTTCAAAGAA } \\
\text { R: TTAACATTGTCTCGGTGTAATCA }\end{array}$ & 149 & 57 & 2 & 0.0000 & 0.4994 & 0.00 \\
\hline N10 & $($ TGT) 12 & $\begin{array}{l}\text { F: CACCTCTCTTGCGGTTGTTT } \\
\text { R: TGTCAAGGAGGACAAGGCTC }\end{array}$ & 118 & 60 & 3 & 0.3077 & 0.4819 & 0.06 \\
\hline N11 & $(\mathrm{ATT})_{5}$ & $\begin{array}{l}\text { F: AACAAGGTTGCCTTCCCATT } \\
\text { R: TAAGACAAGCGTGTGGCAAG }\end{array}$ & 117 & 60 & 2 & 0.0000 & 0.1079 & 0.00 \\
\hline N12 & $(\mathrm{AGA})_{5}$ & $\begin{array}{l}\text { F: CTCCCAATCATTGCCAAATC } \\
\text { R: TATCGCTTCTTGGGCTTCTT }\end{array}$ & 110 & 59 & 2 & 0.3600 & 0.4700 & 0.30 \\
\hline N13 & $(\text { TATT })_{5}$ & $\begin{array}{l}\text { F: CGAATCCATTTGACATCTCTG } \\
\text { R: CGAATGTCGAATCCTAAAGCA }\end{array}$ & 148 & 58 & 2 & 0.3889 & 0.4746 & 0.43 \\
\hline N14 & $(\mathrm{AAAC})_{6}$ & $\begin{array}{l}\text { F: AAGCTGCTCTTCAGTCGCAT } \\
\text { R: TTCTCTCATTCTTCTTCTCTCGC }\end{array}$ & 145 & 59 & 3 & 0.7070 & 0.6435 & 0.16 \\
\hline N15 & $($ TAAT) 5 & $\begin{array}{l}\text { F: AAATCAAATCATCTTGCAAAGC } \\
\text { R: AAACAAAACGGATTGCTTTCT }\end{array}$ & 140 & 57 & 2 & 0.1700 & 0.1760 & 0.60 \\
\hline N16 & $(\mathrm{ATGT})_{9}$ & $\begin{array}{l}\text { F: AATAACACAATCAGTTTATGACAATCA } \\
\text { R: TTGATTTGTTTAAATAGTGTGAAGAGA }\end{array}$ & 113 & 57 & 3 & 0.6522 & 0.6570 & 0.23 \\
\hline N17 & $(\mathrm{AAAG})_{6}$ & $\begin{array}{l}\text { F: GCAAATAGGGGCTGTCAAAC } \\
\text { R: TGAAGTTTCCAAAGCAACGA }\end{array}$ & 106 & 59 & 2 & 0.6000 & 0.4271 & 0.06 \\
\hline N18 & $(\mathrm{AAAT})_{6}$ & $\begin{array}{l}\text { F: CAGCAATTCAAGTTCACCGA } \\
\text { R: CCATTAGCTATTAGCGGTTCTTTT }\end{array}$ & 170 & 59 & 2 & 0.9615 & 0.5090 & 0.00 \\
\hline N19 & $(\mathrm{AGAA})_{5}$ & $\begin{array}{l}\text { F: CTGAGAGCTGTGACACTGATG } \\
\text { R: TGATTTTATGTGTTGCTGGTTG }\end{array}$ & 116 & 57 & 2 & 0.3610 & 0.4720 & 0.30 \\
\hline $\mathrm{N} 20$ & $($ TAAAA) 5 & $\begin{array}{l}\text { F: TCAATATGATAAATTTGACTCTGATGC } \\
\text { R: CCCAAAGTTTCGTCCTATGAT }\end{array}$ & 120 & 58 & 2 & 0.0000 & 0.3638 & 0.00 \\
\hline $\mathrm{N} 21$ & $(\mathrm{AAACA})_{5}$ & $\begin{array}{l}\text { F: TCATTTTCTTTTCACGTTCCC } \\
\text { R: TCAAGCTTCTTCCTTACTATTGCTC }\end{array}$ & 114 & 59 & 3 & 0.5526 & 0.4423 & 0.27 \\
\hline $\mathrm{N} 22$ & $(\mathrm{AAAAT})_{4}$ & $\begin{array}{l}\text { F: TCGACTTCGTCACACGACAT } \\
\text { R: GAGAAAGAAGTGGGCGATGA }\end{array}$ & 115 & 60 & 2 & 0.1176 & 0.2995 & 0.02 \\
\hline $\mathrm{N} 23$ & $\left(\mathrm{AAAAC}_{6}\right.$ & $\begin{array}{l}\text { F: TCTCCCGAGATCTGGTTCAC } \\
\text { R: TTTCAACTTCTCATCGTTCTTGA }\end{array}$ & 136 & 59 & 2 & 0.0417 & 0.3112 & 0.00 \\
\hline $\mathrm{N} 24$ & $($ AAAATC) 5 & $\begin{array}{l}\text { F: GGATTGGAAGGACTGGTGAG } \\
\text { R: TTTTGCTATACCCCGCAAAG }\end{array}$ & 158 & 59 & 2 & 0.1250 & 0.5250 & 0.02 \\
\hline $\mathrm{N} 25$ & $($ CATCAC) 5 & $\begin{array}{l}\text { F: CCATAAGAAGACATGTTTGCCA } \\
\text { R: CGACGAAGACGATAACGACA }\end{array}$ & 101 & 59 & 5 & 0.9333 & 0.6418 & 0.06 \\
\hline N26 & (GATACC) 5 & $\begin{array}{l}\text { F: TGTCATGACTCATCAGCAAGG } \\
\text { R: AATAGTCGGACACCCGATCC }\end{array}$ & 115 & 59 & 2 & 0.0833 & 0.2283 & 0.02 \\
\hline N27 & $\left(\right.$ GAGAAG $_{3}$ & $\begin{array}{l}\text { F: CCAATCAGAAAACGAGAATTGA } \\
\text { R: CAAAATTCGGGAGGCATTTA }\end{array}$ & 170 & 59 & 2 & 0.0000 & 0.3339 & 0.00 \\
\hline N28 & $(\mathrm{AG}){ }_{9} \mathrm{~g}(\mathrm{GA})_{8}$ & $\begin{array}{l}\text { F: ATAGGGTTCACCAATGCAGC } \\
\text { R: TTTTTCCCTGCTTTCACACC }\end{array}$ & 144 & 59 & 2 & 0.2778 & 0.5000 & 0.06 \\
\hline $\mathrm{N} 29$ & $(\mathrm{~A})_{16}$ & $\begin{array}{l}\text { F: GGGTCTCAATCGAATCCTAAAA } \\
\text { R: TTTGCATGACCCAAATTTCA }\end{array}$ & 108 & 59 & 3 & 0.6364 & 0.6480 & 0.31 \\
\hline N30 & $(\mathrm{T})_{17}$ & $\begin{array}{l}\text { F: CTTAGGTGAAGTTCGGCGAC } \\
\text { R: TGAAGAATCTTGTGTGATTTTTCTT }\end{array}$ & 126 & 58 & 3 & 0.0000 & 0.6621 & 0.00 \\
\hline N31 & $(\mathrm{T})_{16}$ & $\begin{array}{l}\text { F: CAACAAAATTTAATCAAACAAACCA } \\
\text { R: AGCAAGTTCTTCTTGCACGG }\end{array}$ & 108 & 59 & 2 & 0.3846 & 0.3167 & 0.25 \\
\hline N32 & (A) 15 & $\begin{array}{l}\text { F: AACCAAAAACTCCAATCCCA } \\
\text { R: CTTCGAAAACCAGTCCGAGA }\end{array}$ & 125 & 59 & 3 & 0.7407 & 0.6744 & 0.15 \\
\hline N33 & $(\mathrm{A})_{17}$ & $\begin{array}{l}\text { F: GTCGCAAGAAGGGCAGATAG } \\
\text { R: TCCAAAACAGAAGAGCCAGG }\end{array}$ & 142 & 59 & 3 & 0.0000 & 0.6079 & 0.00 \\
\hline
\end{tabular}

Continued on next page 
Table 1. Continued.

\begin{tabular}{|c|c|c|c|c|c|c|c|c|}
\hline Locus & Repeat & Primer sequences (5'-3') & Size (bp) & $\mathrm{Ta}\left({ }^{\circ} \mathrm{C}\right)$ & $N_{\mathrm{A}}$ & $H_{\mathrm{O}}$ & $H_{\mathrm{E}}$ & HWE P value \\
\hline N34 & $(\mathrm{T})_{17}$ & $\begin{array}{l}\text { F: GCTAACCTAGTCAGGGCAGG } \\
\text { R: TAGCCGGAATCGAACTCTTG }\end{array}$ & 113 & 58 & 2 & 0.2174 & 0.1981 & 0.60 \\
\hline N35 & $(\mathrm{A})_{18}$ & $\begin{array}{l}\text { F: TGGAGAAGAAATGATGCACAA } \\
\text { R: GGATTGAATTCTCATTATATTTGTTTT }\end{array}$ & 133 & 57 & 3 & 0.5238 & 0.4797 & 0.64 \\
\hline N36 & $(\mathrm{GA})_{13}$ & $\begin{array}{l}\text { F: AAGGGGGAAACTCGGCAG } \\
\text { R: CAAATGCCCAATAACCCAAG }\end{array}$ & 134 & 60 & 3 & 0.0000 & 0.5243 & 0.00 \\
\hline N37 & (TC) 8 & $\begin{array}{l}\text { F: TCAGATCCAACGGCTGTAAA } \\
\text { R: GTTCTCCGATTTTGGGGATT }\end{array}$ & 128 & 59 & 3 & 0.5833 & 0.5771 & 0.86 \\
\hline N38 & $(\mathrm{GA})_{11}$ & $\begin{array}{l}\text { F: CTTACCGGCTACGAGCTACC } \\
\text { R: TTAATGGTCGCTGATACTGAGTT }\end{array}$ & 109 & 57 & 3 & 0.4815 & 0.6730 & 0.12 \\
\hline N39 & $(\mathrm{GA})_{12}$ & $\begin{array}{l}\text { F: AGCCTAATATATAGGGCGAAAAGTG } \\
\text { R: CCAAGGAATTTGTTGCGTG }\end{array}$ & 100 & 60 & 3 & 0.0667 & 0.4994 & 0.00 \\
\hline N40 & $(\mathrm{CT})_{11}$ & $\begin{array}{l}\text { F: CAACCAATTTTCATTACCAAGACA } \\
\text { R: ATTTTTGGAGCCGAATAGCA }\end{array}$ & 149 & 59 & 3 & 1.0000 & 0.6215 & 0.00 \\
\hline N41 & $(\mathrm{AAT})_{5}$ & $\begin{array}{l}\text { F: TTTTGTGAATAGCAAGCAAGC } \\
\text { R: ATAAGGCCCTGGTCTACGGA }\end{array}$ & 153 & 58 & 2 & 0.0000 & 0.5085 & 0.00 \\
\hline N42 & $(\mathrm{ATA})_{5}$ & $\begin{array}{l}\text { F: TGGCACCACATTTCTTCTTTC } \\
\text { R: AAGCCTGCTTCAACCGTAAA }\end{array}$ & 110 & 59 & 3 & 0.1852 & 0.2956 & 0.06 \\
\hline N43 & $(\mathrm{ATA})_{7}$ & $\begin{array}{l}\text { F: CCACTCTGAACTCCTTCTCAGC } \\
\text { R: TAATGGTTGGGGTCATTGGT }\end{array}$ & 117 & 59 & 2 & 0.0000 & 0.4987 & 0.00 \\
\hline N44 & $(\text { TTA })_{5}$ & $\begin{array}{l}\text { F: AACTCCAACGCTAGCGACAC } \\
\text { R: TCAACGAATTAGAGAAAATGAATCC }\end{array}$ & 111 & 59 & 3 & 0.0000 & 0.5672 & 0.00 \\
\hline N45 & $(\mathrm{TCT})_{5}$ & $\begin{array}{l}\text { F: GTCAATGGAAGTTGGAAGGG } \\
\text { R: ATTACTGCGACAGGAGGTCG }\end{array}$ & 114 & 59 & 4 & 0.4091 & 0.4165 & 0.51 \\
\hline N46 & $(\mathrm{TCC})_{6}$ & $\begin{array}{l}\text { F: TCGCAGATCTCCTTTCTATCG } \\
\text { R: CTGTTCCCTTTCATCACGGT }\end{array}$ & 131 & 59 & 2 & 0.4138 & 0.3339 & 0.08 \\
\hline N47 & $(\text { TCTA })_{5}$ & $\begin{array}{l}\text { F: CCATTCACCCTCTCATGGT } \\
\text { R: CCTTTCAGAAGAGGAAGGTTG }\end{array}$ & 146 & 58 & 4 & 0.5833 & 0.6480 & 0.20 \\
\hline N48 & $($ TATG) 4 & $\begin{array}{l}\text { F: ACCAATGATAAACGCCGCTA } \\
\text { R: GCTTATTCCGACTTTTTAACGGT }\end{array}$ & 140 & 59 & 4 & 0.5333 & 0.6751 & 0.15 \\
\hline N49 & $(\mathrm{CTGAT})_{3}$ & $\begin{array}{l}\text { F: ACATGGTTCCACCCAAGG } \\
\text { R: GAGAGGGGTGGTTCTACGGT }\end{array}$ & 104 & 59 & 3 & 0.1333 & 0.5898 & 0.00 \\
\hline N50 & $(\mathrm{AAAAG})_{4}$ & $\begin{array}{l}\text { F: AAAAACAACGGAGTTGACCG } \\
\text { R: TCTTCCTCGCCATTATCGAC }\end{array}$ & 122 & 60 & 4 & 0.5000 & 0.4653 & 0.87 \\
\hline N51 & $(\text { ATAAA) })_{3}$ & $\begin{array}{l}\text { F: TGGTTCAATGTAATTTGGGGA } \\
\text { R: ATACGGTTGTCAACACTGCG }\end{array}$ & 158 & 59 & 3 & 0.4483 & 0.5124 & 0.27 \\
\hline N52 & $(\text { GAAACA })_{3}$ & $\begin{array}{l}\text { F: CCACCACCATCGGAGTAAAG } \\
\text { R: CGCCAACTATAGGCATTAGGA }\end{array}$ & 142 & 59 & 3 & 0.3000 & 0.2695 & 0.84 \\
\hline N53 & $\left(\right.$ ATCAAC $_{3}$ & $\begin{array}{l}\text { F: GACGAGGAGAGCCTTCCAT } \\
\text { R: CCGTTCACCTAATGCACAAA }\end{array}$ & 148 & 59 & 3 & 1.0000 & 0.6153 & 0.00 \\
\hline N54 & $\left(\mathrm{TTGCCC}_{5}\right.$ & $\begin{array}{l}\text { F: TCGCCAGAAGACAAAATTGA } \\
\text { R: TCCGAGGATGAAAAAGGATG }\end{array}$ & 154 & 59 & 3 & 0.0667 & 0.3712 & 0.00 \\
\hline N55 & $(\text { CAGAAA })_{3}$ & $\begin{array}{l}\text { F: GAAACTCTCCTGAGCCATGC } \\
\text { R: TGCTCCCACTTCTGTTTCTG }\end{array}$ & 152 & 59 & 2 & 0.2105 & 0.1935 & 0.66 \\
\hline N56 & $($ AAAAAT) 3 & $\begin{array}{l}\text { F: CCTCCAGAATCCTTGATAGTTCA } \\
\text { R: CATTTGTTTGAATGGTTCATCTG }\end{array}$ & 162 & 59 & 3 & 0.3333 & 0.4983 & 0.24 \\
\hline N57 & $(\text { CAAAAA) })_{3}$ & $\begin{array}{l}\text { F: AGGAGCCCCCAAGTATCAAT } \\
\text { R: TGAGGACGTCATGTTCTCTAGC }\end{array}$ & 141 & 59 & 4 & 0.5517 & 0.6679 & 0.17 \\
\hline N58 & $\left(\right.$ TTTTTA) ${ }_{3}$ & $\begin{array}{l}\text { F: AAATTTGCAATTCTGGTTGTTG } \\
\text { R: TTTCACTCAGCTCGTATCAAACA }\end{array}$ & 113 & 59 & 3 & 0.0000 & 0.4428 & 0.00 \\
\hline N59 & $($ GTGTGG) 4 & $\begin{array}{l}\text { F: TGTGGTGCTTGATACACGGT } \\
\text { R: ATATGACGCCCCACAGATTG }\end{array}$ & 156 & 60 & 2 & 0.2222 & 0.2032 & 0.65 \\
\hline
\end{tabular}

Polymorphism analyses were performed on data from 30 individuals.

To the best of our knowledge, this is the first report about SSR markers of B. tricuspis (Hance) Makino, and it should be useful for genetic analyses and resource conservation in $B$. tricuspis (Hance) Makino.

\section{Conflicts of interest}

The authors declare no conflict of interest.

\section{ACKNOWLEDGMENTS}

Research financially supported by the National Natural Science Foundation of China (Grant 
\#31471547 and \#30900913), the Hunan Provincial Natural Science Foundation of China (Grant \#13JJ4116), the National Modern Agro-industry Technology Research System (Grant \#CARS19-E04), and the National Key Technology Support Program (Grant \#2013BAD01B03-13).

\section{REFERENCES}

Chen JH, Luan MB, Song SF, Zou ZZ, et al. (2011a). Isolation and characterization of EST-SSRs in the Ramie. Afr. J. Microbiol. Res. 5: 3504-3508.

Chen JH, Hu Y, Wang XF, Luan MB, et al. (2011b). Advances in research on germplasms resources in genus Boehmeria Jacq. J. Plant Genet. Resour. 12: 346-351.

LalithaS(2000).PrimerPremier5.BioTechSoftwareInternetReport 1:270-272.http://dx.doi.org/10.1089/152791600459894

Zhang J, Wu YT, Guo WZ and Zhang TZ (2000). Fast screening of microsatellite markers in cotton with page/silver staining. Acta Gossypii Sin. 12: 267-269. 